ARTIGO ORIGINAL ORIGINAL ARTICLE

\section{Internações por Condições Sensíveis à Atenção Primária e indicadores financeiros do SIOPS: uma análise de correlação}

\author{
Hospitalizations for Sensitive Conditions to Primary Care and \\ financial indicators of SIOPS: an analysis of correlation
}

Palloma Lopes de Arruda', Rosana Alves de Melo², Flávia Emília Cavalcante Valença Fernandes³

DOI: $10.21115 / J B E S . v 12 . n 2 . p 142-8$

\section{RESUMO}

Objetivo: Avaliar a correlação dos indicadores financeiros do Sistema de Informações sobre Orçamentos Públicos em Saúde (SIOPS) e Internações por Condições Sensíveis à Atenção Primária (ICSAP) pelos municípios do estado de Pernambuco. Métodos: Trata-se de um estudo ecológico de natureza quantitativa. A população do estudo foi composta pelos 184 municípios do estado de Pernambuco. Foram utilizados dados secundários coletados no TABNET/Datasus e no SIOPS do ano de 2018. Os dados foram analisados inicialmente a partir da estatística descritiva. Foram utilizadas as correlações de Spearman. Resultados: A Receita Líquida Total, a Transferência para a saúde, a Transferência da União para o Sistema Único de Saúde (SUS), a Receita de Transferência SUS e a Despesa Total com Saúde apresentaram influência significativa $(p<0,05)$ quando relacionadas com a taxa de ICSAP. Os outros indicadores do SIOPS não apresentam influência significativa. Conclusão: Os municípios que possuem uma maior receita líquida total possuem menores taxas de ICSAP. Assim, é necessária uma avaliação dos investimentos municipais em saúde para uma priorização da Atenção Primária à Saúde. Dessa forma, aumentando os investimentos e fortalecendo a Atenção Primária, as taxas de ICSAP diminuirão.

\section{Keywords:}

health economics, health expenditures, health status indicators, Primary Health Care, information systems

\begin{abstract}
Objective: Evaluate the correlation of the financial indicators of the Information System on Public Health Budgets (SIOPS) and Hospitalizations for Conditions Sensitive to Primary Care (ICSAP) by the municipalities of the state of Pernambuco. Methods: This is an ecological study of a quantitative nature. The study population consisted of 184 municipalities in the State of Pernambuco. Secondary data collected in TABNET/Datasus and SIOPS in 2018 were used. The data were initially analyzed using descriptive statistics. Spearman's correlations were used. Results: Total Net Revenue, Transfer to health, Transfer from the Union to the Unified Health System (SUS), Revenue from SUS Transfer and Total Health Expense showed significant influence $(p<0.05)$ when related to the rate of ICSAP. The other SIOPS indicators have no significant influence. Conclusion: Municipalities that have a higher total net revenue have lower rates of ICSAP. Thus, it is necessary to evaluate municipal investments in health in order to prioritize Primary Health Care. Thus, increasing investments and strengthening Primary Care, the rates of ACSC will decrease.
\end{abstract}




\section{Introdução}

O conhecimento do financiamento público e do gasto com o Sistema Único de Saúde (SUS) nos municípios demonstra os entraves na organização do sistema e no acesso aos serviços públicos de saúde. Desde a criação do SUS, a falta de estabelecimento de uma fonte de financiamento apropriada e sistemática ocasiona o subfinanciamento do sistema (Santos Neto et al., 2017).

A expansão da Estratégia de Saúde da Família (ESF) impactou positivamente a saúde dos brasileiros, facilitando o acesso aos serviços de saúde com a busca da equidade. Permitiu também uma redução em indicadores de mortalidade como a mortalidade infantil e adulta, inclusive por condições sensíveis à atenção primária, acompanhada de uma diminuição no número de hospitalizações desnecessárias. Sendo assim, os sistemas de saúde que possuem a Atenção Primária à Saúde (APS) no centro de sua organização apresentam resultados mais satisfatórios para a saúde da população (Macinko \& Mendonça, 2018).

Enquanto isso, a efetividade da ESF depende da gestão do cuidado pela equipe multiprofissional, da melhora estrutural dos serviços e da qualificação dos processos organizacionais (Facchini et al., 2018). A redução dos custos com o sistema de saúde depende diretamente da melhora da resolutividade da APS, que inclui a organização do fluxo de encaminhamento dos pacientes para que os serviços de níveis mais elevados da assistência sejam acessados por aqueles que realmente precisarem (Saldiva \& Veras, 2018).

A ausência de equipe completa e especializada na Unidade Básica de Saúde (UBS), a falta de infraestrutura e a inabilidade para o atendimento são alguns dos motivos para o encaminhamento dos pacientes para níveis mais elevados de assistência. Esse fluxo de encaminhamento da APS para serviços mais complexos merece atenção especial, pois, quando acontece de forma desnecessária, o paciente chega a prontos-socorros e hospitais, contribuindo para a superlotação de tais serviços (Saldiva \& Veras, 2018).

As Internações por Condições Sensíveis à Atenção Primária (ICSAP) incluem doenças que podem ser controladas e reduzidas por meio de uma APS resolutiva (Ministério da Saúde, 2008a). As ICSAP permitem medir a qualidade dos serviços de saúde, podendo ser sinalizadoras das ações realizadas na APS. Com isso, a avaliação da efetividade das ações implementadas no território e a identificação dos pontos críticos subsidiam a alteração do perfil de morbimortalidade da população (Maia et al., 2019).

O Sistema de Informações sobre Orçamentos Públicos em Saúde (SIOPS) disponibiliza informações referentes às receitas totais e às despesas com saúde dos orçamentos públicos. Dessa forma, permite o acompanhamento e o monitoramento da alocação de recursos em ações e serviços públicos de saúde, no âmbito da União, estados, Distrito Federal e municípios, auxiliando para a melhoria da gestão. Os indicadores no SIOPS referem-se a: receitas; despesas; dispêndio financeiro; receitas e despesas (Ministério da Saúde, 2008b).

Existem diferentes obstáculos no financiamento e na implantação de políticas para o fortalecimento da APS que demonstram a necessidade de uma análise mais cautelosa dos repasses e alocação dos recursos na saúde. Entre os principais problemas encarados no financiamento da saúde, estão a insuficiência de verba, a ausência de clareza nos parâmetros para os repasses intergovernamentais e a falta de autonomia dos municípios para definir as prioridades relacionadas aos repasses direcionados ao custeio de programas específicos (Silva, 2017).

Desse modo, a vigilância do padrão de financiamento e gasto com saúde pelos municípios auxilia a avaliação da política pública de saúde (Santos Neto et al., 2017). Além disso, o aumento dos investimentos per capita em saúde e a estruturação da ESF promovem a diminuição das ICSAP (Brasil \& Costa, 2016). Então, diminuir os investimentos em sistemas e serviços de saúde pode acarretar níveis mais baixos de qualidade de vida e maiores desigualdades (Macinko \& Mendonça, 2018).

Considerando as políticas públicas de saúde nos níveis nacional, estadual e municipal, especialmente a necessidade de financiamento eficiente para organização e qualificação da APS, espera-se que esse conhecimento possa contribuir com a construção de um SUS de qualidade e uma APS resolutiva. O objetivo deste estudo, portanto, é avaliar a correlação dos indicadores financeiros do SIOPS e ICSAP pelos municípios do estado de Pernambuco.

\section{Métodos}

Trata-se de um estudo ecológico de natureza quantitativa. Esse tipo de estudo tem como unidade de análise uma população ou grupo de pessoas que pertencem a uma área geográfica definida. Avalia ainda como os contextos social e ambiental podem afetar a saúde a partir de uma análise a qual relaciona indicadores de condições de vida e indicadores da situação de saúde (Rouquayrol \& Gurgel, 2013).

A população do estudo foi composta por 184 municípios do estado de Pernambuco. Participaram do estudo os municípios que informaram todos os indicadores utilizados na pesquisa referentes ao ano de 2018, apenas a ilha de Fernando de Noronha foi excluída por não apresentar os dados do SIOPS. De acordo com o art. 75 da Constituição Estadual de 1989, Fernando de Noronha é um Distrito Estadual de Pernambuco, e suas informações orçamentárias são prestadas nesse estado (Assembleia Legislativa do Estado de Pernambuco, 1989).

As informações sobre as ICSAP foram coletadas por meio do Sistema de Informações Hospitalares (SIH) considerando a quantidade de Autorização de Internação Hospitalar (AlH) pagas tabuladas por meio do TABNET/Datasus. As ICSAP, conforme a lista CID-10, incluem: Doenças evitáveis 
por imunização e outras Doenças infecciosas e parasitárias (DIP); Gastroenterites infecciosas e complicações; Anemia; Deficiências nutricionais; Infecções de ouvido, nariz e garganta; Pneumonias bacterianas; Asma; Bronquites; Hipertensão; Angina; Insuficiência cardíaca; Diabetes mellitus; Epilepsias; Infecção no rim e trato urinário; Infecção da pele e tecido subcutâneo; Doença inflamatória nos órgãos pélvicos femininos; Síndrome da Rubéola Congênita.

As informações financeiras foram coletadas no SIOPS por meio dos seguintes indicadores: participação percentual (\%) da receita de impostos na receita total do município, participação percentual (\%) das transferências intergovernamentais na receita total do município, participação percentual (\%) das transferências para a saúde (SUS) no total de recursos transferidos para o município, participação percentual (\%) das transferências da União para a saúde (SUS) no total de transferências da União para o município, participação percentual (\%) da despesa com investimentos na despesa total com saúde, participação percentual (\%) da transferência para a saúde (SUS) na despesa total com saúde, participação percentual (\%) da receita própria aplicada em saúde conforme a Emenda Constitucional (EC) no 29/2000, instituída pela Constituição Federal de 1988 (CF/88) e regulamentada pela Lei Complementar (LC) no 141/2012, e despesa total com saúde.

As internações foram analisadas de duas formas, inicialmente por meio da frequência absoluta das $\mathrm{AlH}$. Posteriormente, foi calculada a taxa de ICSAP por região de saúde, onde o numerador foi a frequência de ICSAP (representada pelo quantitativo de AlH pagas) dividido pela estimativa populacional de cada região, multiplicado por 100.000 . A fase seguinte foi de análise dos dados, iniciando-se com a estatística descritiva por meio das medidas de tendência central e dispersão.

Foram utilizadas as correlações de Spearman, considerando a não normalidade da distribuição testada pelo ShapiroWilk. O sentido da correlação foi verificado pelo sinal do coeficiente rho. Para todos os testes, foram adotadas confiança de 95\% e significância de 5\%. Foram utilizados o software Microsoft Office Excel 2013 e o programa estatístico Stata 14.0.

Esta pesquisa obedeceu aos preceitos estabelecidos na Resolução no 510, de 7 de abril de 2016, do Conselho Nacional de Saúde (Brasil, 2016). Por se tratar de estudo com dados secundários, não foi necessária a submissão ao Comitê de Ética e Pesquisa nem a utilização do Termo de Consentimento Livre e Esclarecido.

\section{Resultados}

A média de AlH pagas por ICSAP encontrada entre os 184 municípios do estado de Pernambuco foi de 332, apresentando disparidade entre os municípios (DP 978). O valor médio da Receita Líquida Total foi de 4,3\% e o de Transferências Intergovernamentais Líquidas, de 83,9\%. A média de Transferência de recursos para a saúde (SUS) do total transferido para o município foi de 13,4\%, enquanto a Transferência da União para o SUS obteve 22,9\%.

Quanto à Despesa com investimentos pela Despesa Total, obteve-se uma média de 6,8\%. O percentual de despesas com saúde financiadas com recursos transferidos por outras esferas de governo representado pelo indicador Transferência SUS pela Despesa Total apresentou uma média de 54,0\%. Os Recursos Próprios em Saúde (EC no 29), que representam o cumprimento do percentual mínimo aplicado em Ações e Serviços Públicos de Saúde (ASPS) pelo município, obtiveram uma média de 20,3\%. A Despesa Total com Saúde correspondeu a uma média de R\$27.000.000 (Tabela 1).

Quanto à média de ICSAP por Região de Saúde, a I Região alcançou maior média (1.558), apresentando também a maior Receita Líquida Total, com 10,6\% de média. A Transferência para a saúde (SUS) e a Transferência da União para a saúde (SUS) obtiveram maior média na II Região de 14,9\% e na I Região de 27,6\%, respectivamente (Tabela 2).

Tabela 1. Distribuição das Internações por Causas Sensíveis à Atenção Primária e indicadores do SIOPS segundo municípios Pernambuco, Brasil, 2018

\begin{tabular}{|c|c|c|c|c|c|c|}
\hline Variável & Média & $\begin{array}{l}\text { Desvio-padrão } \\
\text { (DP) }\end{array}$ & Mínimo & Máximo & \multicolumn{2}{|c|}{ IC $95 \%$} \\
\hline AlH pagas por Causas Sensíveis à Atenção Primária & 332 & 978 & 14 & 11.708 & 191,9 & 477,2 \\
\hline Receita Líquida Total (\%) & 4,3 & 4,3 & 0,7 & 33,5 & 3,7 & 4,9 \\
\hline Transferências Intergovernamentais Líquidas (\%) & 83,9 & 8,0 & 50,1 & 97,6 & 82,7 & 85,0 \\
\hline Transferência para a saúde (SUS) (\%) & 13,4 & 3,8 & 2,6 & 25,2 & 12,8 & 13,9 \\
\hline Transferência da União para o SUS (\%) & 22,9 & 6,5 & 8,4 & 49,6 & 21,9 & 23,4 \\
\hline Despesa Investimentos/Despesa Total (\%) & 6,8 & 5,0 & 0,0 & 30,7 & 6,0 & 7,5 \\
\hline Transferência SUS/Despesa Total (\%) & 54,0 & 12,3 & 9,7 & 93,1 & 52,3 & 55,8 \\
\hline Recursos Próprios em Saúde (EC n²9) (\%) & 20,3 & 4,9 & 11,8 & 34,9 & 19,6 & 21,0 \\
\hline Despesa Total com Saúde (R\$ em milhões) & 27,0 & 89,0 & 0,0 & 1.150 & 14,2 & 40,1 \\
\hline
\end{tabular}


A Despesa com Investimento pela Despesa Total foi maior na VII Região, apresentando média de 10,0\%. A Transferência SUS pela Despesa Total apresentou média de 59,4\% na IX Região. Os Recursos Próprios em Saúde (EC n²9) obtiveram maior média na VIII Região, com 26,2\%, e a Despesa Total com Saúde apresentou maior média na I Região (R\$ 125.000.000) (Tabela 3).

Quanto à taxa de ICSAP segundo a Região, observou-se uma maior média na VII Região (852,35/100.000 habitantes) (Tabela 4).

A Receita Líquida Total, a Transferência para a saúde (SUS), a Transferência da União para a saúde (SUS) e a
Despesa Total com Saúde apresentaram correlação significativa $(p<0,05)$ quando relacionadas com a taxa de ICSAP. Contudo, o sentido das correlações foi diferente. Quanto maior o percentual de receita líquida total, menor foi a taxa de ICSAP. Entretanto, maiores proporções de transferência para a saúde (SUS), maiores as transferências da União para o SUS e maiores as Despesas Totais com saúde e maiores também foram as taxas de ICSAP. Os outros indicadores do SIOPS não apresentam influência significativa $(p>0,05)$ (Tabela 5).

Tabela 2. Distribuição das Internações por Causas Sensíveis à Atenção Primária e indicadores do SIOPS segundo Região de Saúde Pernambuco, Brasil, 2018

\begin{tabular}{|c|c|c|c|c|c|c|c|c|c|c|c|c|}
\hline \multirow{3}{*}{$\begin{array}{c}\begin{array}{c}\text { Região } \\
\text { de Saúde }\end{array} \\
\text { I }\end{array}$} & \multicolumn{3}{|c|}{ AlH } & \multicolumn{3}{|c|}{ \% Receita Líquida Total } & \multicolumn{3}{|c|}{$\begin{array}{l}\text { \% Transferência } \\
\text { para a saúde (SUS) }\end{array}$} & \multicolumn{3}{|c|}{$\begin{array}{l}\text { \% Transferência da } \\
\text { União para o SUS }\end{array}$} \\
\hline & \multirow{2}{*}{$\frac{\text { Média }}{1.558}$} & \multicolumn{2}{|c|}{ IC 95\% } & \multirow{2}{*}{$\frac{\text { Média }}{10,6}$} & \multicolumn{2}{|c|}{ IC 95\% } & \multirow{2}{*}{$\begin{array}{c}\text { Média } \\
13,4\end{array}$} & \multicolumn{2}{|c|}{ IC 95\% } & \multirow{2}{*}{$\begin{array}{c}\text { Média } \\
27,6\end{array}$} & \multicolumn{2}{|c|}{ IC $95 \%$} \\
\hline & & 323,8 & 2791,3 & & 6,8 & 14,3 & & 11,1 & 15,7 & & 23,5 & 31,7 \\
\hline$\|$ & 159 & 105,4 & 213,0 & 3,0 & 2,3 & 3,8 & 14,9 & 13,3 & 16,5 & 24,0 & 21,4 & 26,5 \\
\hline III & 151 & 99,7 & 202,8 & 3,2 & 1,9 & 4,5 & 13,3 & 11,4 & 15,3 & 22,1 & 19,2 & 25,0 \\
\hline IV & 210 & 116,0 & 304,2 & 4,4 & 3,1 & 5,7 & 13,3 & 12,0 & 14,5 & 22,1 & 19,9 & 24,3 \\
\hline V & 152 & 80,0 & 224,0 & 2,8 & 1,9 & 3,7 & 14,2 & 13,3 & 15,2 & 24,1 & 22,3 & 25,8 \\
\hline VI & 273 & 106,5 & 440,1 & 4,4 & 2,6 & 6,2 & 12,7 & 10,4 & 15,1 & 20,6 & 17,2 & 24,1 \\
\hline VII & 195 & 45,3 & 345,6 & 3,6 & 0,7 & 6,5 & 11,2 & 9,4 & 12,9 & 18,4 & 14,5 & 22,2 \\
\hline VIII & 365 & $-91,3$ & 820,7 & 5,3 & 2,1 & 8,4 & 13,3 & 11,6 & 15,0 & 22,9 & 18,3 & 27,5 \\
\hline IX & 268 & 55,0 & 481,4 & 3,0 & 2,6 & 3,5 & 14,1 & 12,5 & 15,6 & 24,1 & 21,4 & 26,9 \\
\hline$x$ & 90 & 29,9 & 150,8 & 3,3 & 2,6 & 4,0 & 12,5 & 10,4 & 14,6 & 20,8 & 17,7 & 24,0 \\
\hline$X I$ & 230 & $-12,5$ & 471,7 & 3,8 & 2,2 & 5,4 & 12,0 & 9,4 & 14,6 & 19,9 & 15,4 & 24,5 \\
\hline XII & 173 & 86,2 & 259,2 & 3,3 & 2,2 & 4,5 & 13,4 & 11,1 & 15,6 & 23,4 & 20,6 & 26,1 \\
\hline
\end{tabular}

Tabela 3. Distribuição dos indicadores do SIOPS segundo Região de Saúde - Pernambuco, Brasil, 2018

\begin{tabular}{|c|c|c|c|c|c|c|c|c|c|c|c|c|}
\hline \multirow{3}{*}{$\begin{array}{c}\begin{array}{c}\text { Região } \\
\text { de Saúde }\end{array} \\
\mid\end{array}$} & \multicolumn{3}{|c|}{$\begin{array}{c}\text { \% Despesa Investimento/ } \\
\text { Despesa Total }\end{array}$} & \multicolumn{3}{|c|}{$\begin{array}{c}\text { \% Transferência SUS/ } \\
\text { Despesa Total }\end{array}$} & \multicolumn{3}{|c|}{$\begin{array}{l}\text { \% Recursos Próprios } \\
\text { em Saúde (EC n²9) }\end{array}$} & \multicolumn{3}{|c|}{$\begin{array}{l}\text { Despesa Total com Saúde } \\
\text { (R\$ em milhões) }\end{array}$} \\
\hline & \multirow{2}{*}{$\begin{array}{c}\text { Média } \\
3,1\end{array}$} & \multicolumn{2}{|c|}{ IC 95\% } & \multirow{2}{*}{$\begin{array}{c}\text { Média } \\
48,3\end{array}$} & \multicolumn{2}{|c|}{ IC 95\% } & \multirow{2}{*}{$\begin{array}{c}\text { Média } \\
18,3\end{array}$} & \multicolumn{2}{|c|}{ IC 95\% } & \multirow{2}{*}{$\begin{array}{r}\text { Média } \\
125,0\end{array}$} & \multicolumn{2}{|c|}{ IC 95\% } \\
\hline & & 1,9 & 4,2 & & 41,6 & 55,1 & & 17,1 & 19,5 & & 8,7 & 242,0 \\
\hline$\|$ & 8,8 & 5,5 & 12,1 & 57,9 & 52,9 & 62,9 & 22,1 & 19,5 & 24,8 & 15,1 & 11,2 & 19,0 \\
\hline III & 5,3 & 3,4 & 7,2 & 57,1 & 50,9 & 63,4 & 19,3 & 17,5 & 21,1 & 13,7 & 10,4 & 16,9 \\
\hline IV & 6,8 & 5,1 & 8,5 & 55,9 & 51,9 & 59,8 & 19,7 & 18,4 & 21,1 & 20,2 & 9,3 & 31,0 \\
\hline V & 7,7 & 5,7 & 9,7 & 56,2 & 51,7 & 60,6 & 19,0 & 17,0 & 21,0 & 14,3 & 9,3 & 19,4 \\
\hline $\mathrm{Vl}$ & 5,6 & 3,3 & 7,9 & 48,4 & 42,3 & 54,6 & 22,7 & 19,5 & 25,9 & 15,7 & 12,0 & 19,4 \\
\hline VII & 10,0 & 5,3 & 14,7 & 42,8 & 38,0 & 47,6 & 21,7 & 17,9 & 25,5 & 11,5 & 6,4 & 16,7 \\
\hline VIII & 6,2 & 4,4 & 8,0 & 50,9 & 44,2 & 57,6 & 26,2 & 20,8 & 31,5 & 31,4 & $-1,5$ & 64,4 \\
\hline IX & 9,3 & 6,0 & 12,5 & 59,4 & 54,3 & 64,4 & 22,1 & 18,5 & 25,8 & 15,4 & 9,4 & 21,4 \\
\hline$x$ & 6,4 & 4,5 & 8,3 & 52,8 & 46,0 & 59,7 & 18,5 & 16,9 & 20,1 & 9,23 & 6,2 & 12,2 \\
\hline$X I$ & 9,0 & 6,0 & 12,1 & 49,5 & 41,4 & 57,6 & 19,4 & 17,2 & 21,5 & 13,1 & 6,0 & 20,1 \\
\hline XII & 6,2 & 4,0 & 8,5 & 57,6 & 50,5 & 64,6 & 20,5 & 17,1 & 23,9 & 15,1 & 8,9 & 21,4 \\
\hline
\end{tabular}


Tabela 4. Taxa de Internações por Condições Sensíveis à Atenção Primária segundo Região de Saúde - Pernambuco, Brasil, 2018

\begin{tabular}{|c|c|c|c|}
\hline Região de Saúde & Média & & \\
\hline I & 679,10 & 574,30 & 783,90 \\
\hline$\|$ & 536,99 & 460,33 & 613,65 \\
\hline III & 513,40 & 444,47 & 582,32 \\
\hline IV & 543,63 & 451,90 & 635,37 \\
\hline V & 631,29 & 546,21 & 716,37 \\
\hline $\mathrm{VI}$ & 713,70 & 467,06 & 960,35 \\
\hline VII & 852,35 & 711,24 & 993,45 \\
\hline VIII & 549,04 & 402,48 & 695,60 \\
\hline IX & 703,00 & 512,61 & 893,39 \\
\hline$x$ & 469,08 & 341,70 & 596,47 \\
\hline$X I$ & 754,94 & 544,44 & 965,44 \\
\hline$X I I$ & 533,24 & 442,16 & 624,33 \\
\hline
\end{tabular}

Tabela 5. Correlações entre a taxa de Internações por Condições Sensíveis à Atenção Básica e indicadores do SIOPS - Pernambuco, Brasil, 2018

\begin{tabular}{llc}
\hline Variável & rho & p-valor* \\
\hline Receita Líquida Total (\%) & $-0,188$ & 0,011 \\
\hline Transferências Intergovernamentais Líquidas (\%) & 0,113 & 0,126 \\
\hline Transferência para a saúde (SUS) (\%) & 0,149 & 0,044 \\
\hline Transferência da União para o SUS (\%) & 0,004 \\
\hline Despesa Investimentos/Despesa Total (\%) & $-0,032$ & 0,672 \\
\hline Transferência SUS/Despesa Total (\%) & 0,012 & 0,869 \\
\hline Recursos Próprios em Saúde (EC no 29) (\%) & 0,399 \\
\hline Despesa Total com Saúde & $-0,063$ & 0,012 \\
\hline
\end{tabular}

* Correlação de Spearman

\section{Discussão}

A média de AlH pagas por causas sensíveis à atenção primária apresentou disparidades entre os municípios na análise realizada, assim como a taxa entre as regiões de saúde. De acordo com Departamento de Saúde da Família (DESF), o estado de Pernambuco apresenta uma cobertura de ESF de $76,71 \%$, então essa disparidade na média de $\mathrm{AlH}$ pode estar relacionada à diferença de cobertura da ESF entre os municípios (Brasil, 2019).

O valor médio da Receita Líquida Total que dimensiona a capacidade de arrecadação do município (Brasil, 2009) foi baixo no presente estudo. Entretanto, quanto maior foi o percentual de receita líquida total, menor foi a taxa de ICSAP. Tal resultado pode mostrar que municípios com maiores arrecadações dependem menos de outras esferas e, consequentemente, conseguem ter menores taxas de internação, podendo oferecer uma APS mais eficiente. Sendo assim, possuem maiores condições de custear as necessidades de saúde de seus munícipes (Simão \& Orellano, 2015). Para o financiamento municipal da saúde, a receita própria tem grande importância, a partir do momento em que o acréscimo da despesa com saúde está mais relacionado à arrecadação municipal do que às transferências intergovernamentais (Feliciano et al., 2017).

No que diz respeito às Transferências Intergovernamentais Líquidas que dimensionam a dependência do município em relação aos recursos transferidos pela União e pelos estados (Brasil, 2009), a média foi alta. Esses resultados concordam com o estudo realizado por Teixeira e Teixeira (2003), no qual $65 \%$ da receita total dos municípios foi resultante da transferência de recursos de outras esferas. O modo de financiamento do SUS trouxe um aumento na fragmentação das transferências, dificultando a descentralização e a autonomia dos municípios, por isso não facilitou o processo de regionalização do SUS (Duarte et al., 2018).

Quanto maior for o percentual de Transferência de recursos para a saúde (SUS), maiores serão as transferências específicas para a saúde em relação ao total de transferências 
feitas pela União ao município (Brasil, 2009). Isso mostra a dependência dos municípios em relação à União, e questiona-se até onde o município consegue atender ao princípio da universalidade com essa insuficiência de recursos para gerir a saúde em sua extensão territorial (Feliciano et al., 2017).

Os municípios de Pernambuco apresentaram uma média baixa de Transferência de recursos para a saúde (SUS), demonstrando quanto dos recursos recebidos pelo município, por meio de transferências, é direcionado para a saúde (Brasil, 2009). No entanto, sobre a Transferência da União para o SUS, a média foi maior, reforçando a dependência dos municípios sobre a União. O impacto desse cenário pode ser observado no presente estudo, o qual mostrou que maiores dependências de outros recursos, analisados pelos indicadores transferência para a saúde (SUS), transferências da União para o SUS e Despesas Totais com Saúde, geram maiores taxas de ICSAP e também que quanto maiores foram esses indicadores, maiores também foram as taxas.

Sobre o indicador Investimentos em Saúde, o qual indica o nível dos investimentos realizados pelo município em saúde (Brasil, 2009), apresentou uma média baixa. Estudo realizado com os municípios de São Paulo mostrou que o maior percentual de gasto com investimentos em saúde foi de $4,45 \%$. Esse percentual muito baixo comprova que a gestão municipal está direcionando poucos recursos para a compra de bens permanentes, para a construção de unidades novas ou para a ampliação das unidades já existentes (Santos Neto et al., 2017).

Mais da metade da despesa total com saúde dos municípios estudados no presente artigo vem de outras esferas, observadas pelo indicador Transferência SUS pela Despesa Total. No processo de descentralização do sistema de saúde, é importante acompanhar as transferências e o comportamento das instâncias subnacionais em relação aos gastos públicos para, assim, organizar a administração pública (Feliciano et al., 2019).

O presente estudo evidenciou que os municípios cumprem o instituído pela CF/88 e regulamentado pela LC no 141/2012 sobre o percentual mínimo aplicado em Ações e Serviços Públicos de Saúde (ASPS), estando acima do estabelecido. Esse indicador dimensiona o nível de aplicação dos impostos e transferências dada à obrigatoriedade de se aplicar o limite mínimo de 15\% em ASPS (Brasil, 2009). Percentual semelhante foi encontrado em pesquisa realizada no estado de São Paulo, onde os recursos próprios do município aplicados em saúde apresentaram uma média de 23,8\%, também acima do percentual mínimo de 15\% definido pela EC no 29 (Soares, 2019).

A Despesa Total com Saúde, incluindo a financiada por outras esferas, por habitante, foi incluída no presente estudo, apresentando uma média baixa. Pesquisa realizada com os municípios da Bahia mostrou que cerca de 26\% (107 municípios) encontraram-se com uma despesa na faixa de R\$ 6 a 25 milhões, corroborando o resultado do presente estudo, que encontrou uma média de R\$ 27 milhões (Teles et al., 2017). Ademais, quanto maiores foram as despesas totais com saúde, maiores também foram as taxas de internação por condições sensíveis à APS.

Quando se relaciona a política de saúde ao porte populacional, essa confere diferentes requisitos, desde a complexidade até a classificação dos serviços, dependendo da quantidade de habitantes (Araújo et al., 2017). Levando em conta a produtividade dos municípios, visualiza-se a diferença entre eles, inclusive dentro da mesma região de saúde (Campos \& Gurgel, 2016). Além disso, o processo de regionalização apresenta desafios como a deficiência na estrutura das regionais de saúde, desde o desenho dos serviços ofertados na rede até a integração (Viana et al., 2017).

Por isso, em metade dos indicadores, a I Região de Saúde apresentou os valores mais altos nas médias por região de saúde. O porte populacional, a complexidade dos serviços ofertados e a centralização de alguns serviços nessa região podem justificar esses indicadores.

\section{Conclusão}

Evidenciou-se que o percentual da receita líquida é inversamente proporcional à taxa de internação. Os principais indicadores financeiros do SIOPS que estiveram correlacionados com as ICSAP foram as proporções de Transferência para a saúde (SUS), Transferências da União para o SUS e Despesas Totais com Saúde. Desses, observou-se que os municípios que mais gastaram com saúde não necessariamente foram os que apresentaram maiores ICSAP. Além disso, os municípios com maiores dependências de outros recursos apresentaram maiores taxas de internação.

Dessa forma, evidencia-se que os indicadores financeiros têm o potencial de influenciar na resolutividade da APS, aumentando sua eficiência e implicando uma menor demanda para outros níveis de assistência onde os custos são mais elevados, o que pode impactar diretamente na redução das internações por condições sensíveis aos problemas existentes na APS. Assim, vê-se uma diminuição das taxas de ICSAP caso haja o fortalecimento e o aumento dos investimentos na APS, sendo essa uma das alternativas que se mostra mais importante para a diminuição dos gastos nesse segmento.

Entre as limitações do estudo, estão a forma de alimentação dos Sistemas de Informação em Saúde, que depende inteiramente dos municípios, e o fato de a pesquisa ter sido realizada com dados de apenas um ano, necessitando de novos estudos que possam abranger um maior intervalo de tempo, de forma a ampliar o leque de discussão utilizando os dados existentes.

\section{Referências bibliográficas}

Araújo CE, Gonçalves GQ, Machado JA. Os municípios brasileiros e os gastos próprios com saúde: algumas associações. Ciênc Saúde Coletiva. 2017;22(3):953-63. 
Assembleia Legislativa do Estado de Pernambuco (ALEPE). Constituição do Estado de Pernambuco de 1989.

Brasil. Ministério da Saúde. Departamento de Saúde da Família (DESF). 2019. Available from: https://egestorab.saude.gov.br/.

Brasil. Ministério da Saúde. Portaria MS/SAS n 221, de 17 de abril de 2008a.

Brasil. Ministério da Saúde. NOTA TÉCNICA No 58 /2008b - SIOPS/AESD/SE/ MS. Avaiable from: http://siops.datasus.gov.br/Documentacao/NT\%20 -\%20Revis\%C3\%A30\%20dos\%20Indicadores\%20.pdf.

Brasil. Ministério da Saúde. NOTA TÉCNICA 2009 - SIOPS/AESD/SE/MS, DE

23/03/2009. Avaliable from: http://siops.datasus.gov.br/Documentacao/ NT\%20apresenta\%C3\%A7\%C3\%A30\%20Indicadores\%20-\%20a\%20 partir\%20do\%201\%C2\%BA\%20semestre\%20de\%20\%202007.pdf.

Brasil, Resolução Conselho Nacional de Saúde (CNS) n 510, de 7 de abril de 2016. Available from: http://bit.ly/2fmnKeD.

Brasil VP, Costa JS. Hospitalizações por condições sensíveis à atenção primária em Florianópolis, Santa Catarina - estudo ecológico de 2001 a 2011. Epidemiol Serv Saúde. 2016;25(1):75-84.

Campos AG, Gurgel AM. Acidentes de trabalho graves e atividades produtivas nas regiões administrativas de saúde em Pernambuco: uma análise a partir da identificação de aglomerados produtivos locais. Rev Bras Saúde Ocup. 2016;41(e15):1-12.

Duarte LS, Mendes AN, Louvison MC. O processo de regionalização do SUS e a autonomia municipal no uso dos recursos financeiros: uma análise do estado de São Paulo (2009-2014). Saúde Debate. 2018;42(116):25-37.

Facchini LA, Tomasi E, Dilélio AS. Qualidade da Atenção Primária à Saúde no Brasil: avanços, desafios e perspectivas. Saúde Debate. 2018;42(1):208-23.

Feliciano M, Bezerra AF, Santo AC. Crescimento econômico e taxa de inflação: implicações na receita municipal e na despesa com saúde dos municípios de Pernambuco, Brasil. Ciênc Saúde Coletiva. 2017;22(6):1979-90.

Feliciano M, Medeiros KR, Damázio SL, Alencar FL, Bezerra AF. Avaliação da cobertura e completitude de variáveis de Sistemas de Informação sobre orçamentos públicos em saúde. Saúde Debate. 2019;43(121):341-53.
Macinko J, Mendonça CS. Estratégia Saúde da Família, um forte modelo de Atenção Primária à Saúde que traz resultados. Saúde Debate. 2018;42(1):18-37.

Maia LG, Silva LA, Guimarães RA, Pelazza BB, Pereira AC, Rezende WL, et al. Internações por condições sensíveis à atenção primária: um estudo ecológico. Rev Saúde Pública. 2019;53(2):1-11.

Rouquayrol MZ, Gurgel M. Epidemiologia \& Saúde. 7a ed. Rio de Janeiro: Medbook; 2013.

Saldiva PH, Veras M. Gastos públicos com saúde: breve histórico, situação atual e perspectivas futuras. Estud Av. 2018;32(92):47-61.

Santos Neto JA, Mendes ÁN, Pereira AC, Paranhos LR. Análise do financiamento e gasto do Sistema Único de Saúde dos municípios da região de saúde Rota dos Bandeirantes do estado de São Paulo, Brasil. Ciênc Saúde Coletiva. 2017;22(4):1269-80.

Silva IB. Desafios do financiamento da atenção primária à saúde: revisão integrativa. Rev Bras Promoção Saúde. 2017;30(1):110-7.

Simão JB, Orellano VI. Um estudo sobre a distribuição das transferências para o setor de saúde no Brasil. Estud Econ. 2015;45(1):33-63.

Soares A. Paradigma de financiamento do SUS no estado de São Paulo: uma análise regional. Rev Saúde Pública. 2019;53(39):1-11.

Teixeira HV, Teixeira MG. Financiamento da saúde pública no Brasil: a experiência do SIOPS. Ciênc Saúde Coletiva. 2003;8(2):379-1.

Teles AS, Coelho TC, Ferreira MP. Gasto Público em Saúde na Bahia: explorando indícios de desigualdades. Saúde Debate. 2017;41(113):457-70.

Viana AL, Ferreira MP, Cutrim MA, Fusaro ER, Souza MR, Mourão L, et al. 0 Processo de Regionalização no Brasil: influência das dimensões Política, Estrutura e Organização. Rev Bras Saude Mater Infant. 2017;17(Supl 1):S45-61. 